Yi Sun* and Xinping Sun

\title{
Exploring the interstitial system in the brain: the last mile of drug delivery
}

https://doi.org/10.1515/revneuro-2020-0057

Received June 27, 2020; accepted December 8, 2020; published online January 27,2021

\begin{abstract}
Brain interstitial system (ISS) is a nanoscale network of continuously connected tubes and sheets surrounding each neural cell in the central nervous system. ISS usually accounts for $\sim 20 \%$ of the brain volume, far more than the cerebral blood vessels, which account for $3 \%$. The neuronal function, signaling pathways, and drug delivery are all closely related to the microenvironment provided by ISS. The objective of this paper is to give the readers a clear outline of detection, anatomy, function, and applications of ISS. This review describes the techniques propelling the exploration for ISS in chronological order, physiological function and pathological dysfunction of ISS, and strategies for drug delivery based on ISS. Biophysical features are the focus of ISS research, in which the diffusion characteristics have dominated. The various techniques that explore ISS take advantage of this feature. ISS provides an essential microenvironment for the health of cells and brain homeostasis, which plays an important functional role in brain health and disease. Direct intracranial administration allows the diffusion of drugs directly through ISS to successfully bypass the blood-brain barrier that prevents most drugs from reaching the brain. With the deepening of understanding of the brain ISS, the new research model that takes into account brain cells, cerebral vessels, and ISS will provide a new perspective and direction for understanding, utilizing, and protecting the brain.
\end{abstract}

Keywords: diffusion characteristics; drug delivery; interstitial system; (patho)physiological functions; superresolution fluorescence microscopy.

Yi Sun and Xinping Sun contributed equally to this article.

*Corresponding author: Yi Sun, National Key Research Laboratory of Natural and Biomimetic Drugs, Peking University, Beijing 100191, P. R. China; and Department of Molecular and Cellular Pharmacology, School of Pharmaceutical Sciences, Peking University, Beijing 100191, P. R. China, E-mail: sunyi@bjmu.edu.cn

Xinping Sun, Clinical Laboratory, Peking University International Hospital, Beijing 102206, P. R. China

\section{Introduction}

Brain interstitial system (ISS) is a nanoscale network of continuously connected tubes and sheets surrounding each brain cell; the space between adjacent neural cells is also known as extracellular space (ECS), filled with mesenchymal fluid (ISF) and extracellular matrix (ECM), which dissolves a variety of the substances needed for brain cell function, including metal ions, nutrients, hormones, and neurotransmitters (Figure 1). Meanwhile, the ISS is also a key microenvironment for the removal of toxic metabolites. Despite being only $20-60 \mathrm{~nm}$ in width, the ISS typically occupies $15-30 \%$ of the brain volume (Lehmenkuhler et al. 1993; Sykova and Nicholson 2008; Van Harreveld and Steiner 1970). Therefore, the ISS should not be neglected, due to its important contents and its considerable volume fraction $(\alpha)$ in the brain. Over the last 60 years, as some important functions of the extracellular space continue to be discovered, the ISS has been in the center of the researchers' interest. One of the most exciting findings was that the $\alpha$ and the metabolic clearance of ISS changed dramatically during the sleep-wake cycle (Ding et al. 2016), suggesting its active regulatory role in brain physiology. We know far less about ISS than we do about the two main compartments of the brain, neurons, and glial cells, which is largely limited by research techniques. These channels' width is estimated at a scale of tens of nanometers which is smaller than an optical microscope's resolution; however, it is eliminated by most traditional electron microscope procedures. As a result, the limited knowledge regarding the ISS has urged experts of the field to investigate it, thus emerging new research methods. Therefore, this article will review the arduous process of exploring ISS.

\section{With the advent of new research techniques, the mystery of ISS is being revealed}

The exploration of the ISS focuses on two aspects: geometric structure imaging and the estimation of physical parameters related to the structural characteristics under physiological and pathological conditions. Most of our 


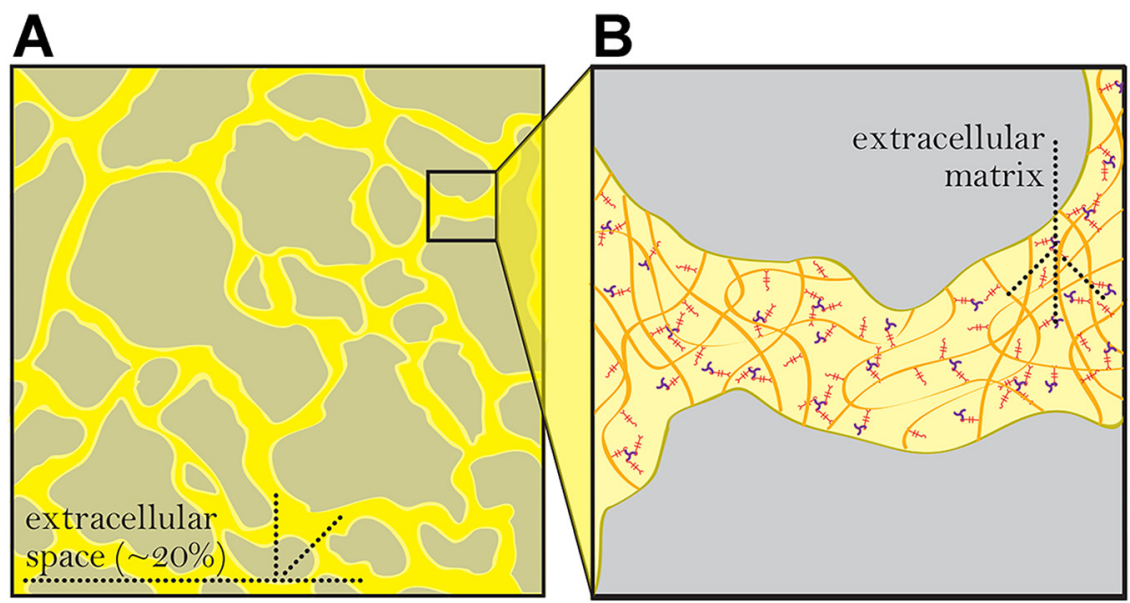

Figure 1: Diagram of ISS. (A) The geometry of ISS in a typical brain section. Yellow marks the ISS between the gray brain cell processes. The volume fraction of the ISS is roughly $20 \%$ under physiological conditions. (B) Enlarged image of the black box areas in (A). Highlights physical parameters contributing to ISS diffusion characteristics, including brain cell geometry (gray) and ECM (diagramed as a mesh of multicolored glycosaminoglycans and proteoglycans). Figure modified from Odackal et al. (2017). understanding of ISS in brain tissue is derived from molecular diffusion studies, which infer the structural parameters of ISS through the diffusion of extracellular probe molecules (Saghyan et al. 2012). At each stage of technological development, multiple methods exist simultaneously. Therefore, this review will cover only the representative ones.

\section{The beginning of ISS exploration}

Prior to the imaging of the brain's ultrastructure, measurements of diffused ion concentration had shown that ISS accounted for approximately $20 \%$ of the total volume of brain tissue. The method of direct diffusion measurements is to introduce detectable substances into ISS and measure the distribution of their concentrations in space and time (Sykova and Nicholson 2008). As far back as the 1950s, Allen estimated the ISS of rat brain through an in vitro diffusion technique with the use of sodium ferrocyanide and inulin, which does not penetrate the living cells of the nerve tissue. In the experiment, acute slices of rat brains were incubated in an isotonic solution containing the two compounds. Following the allowance for diffusion and equilibration, the concentration of the test substance was determined in both brain slices and the external medium. The space of diffusion could then be calculated. The values obtained are 17 and $14.5 \mathrm{ml}$ per $100 \mathrm{gm}$, respectively (Allen 1955). The tissue distribution space was calculated by the equation

$$
\text { Space }(\mathrm{ml} / 100 \mathrm{gm})=\frac{C_{\tau}(\mathrm{mg} / \mathrm{gm})}{C_{m}(\mathrm{mg} / \mathrm{ml})} \times 100
$$

where $C_{t}$ is the equilibrium concentration in tissue and $C_{m}$ is the concentration in the medium. Today, the volume fraction is denoted by $\alpha$. This experiment solved a rather complex problem in a pretty simple way. Furthermore, the results were confirmed by various in vivo experiments using subsequently developed resistivity measurements and diffusion analysis.

More than a decade later, Fenstermacher and colleagues established the workable in vivo radiotracer technique. In short, the method was to stereotaxically perfuse the subarachnoid space of an anesthetized animal with a radiotracer, $\left[{ }^{3} \mathrm{H}\right]$-inulin and $\left[{ }^{14} \mathrm{C}\right]$-sucrose, for $2-3 \mathrm{~h}$. After perfusion, the brain was rapidly removed, frozen, and cut into a series of coronal sections. Rectangular strips of cortical tissue were cut perpendicular to the surface and then serially sectioned into either 0.5 or $1.0 \mathrm{~mm}$ thick slices by a single stroke of a special multibladed knife (Figure 2). Each section was weighed and the radioactivity measured to obtain a profile of the concentration of the radiotracer at different distances from the surface at the time the animal was sacrificed. The tissue distribution space was calculated by the equation similar to Allen's

$$
\text { Space }(\%)=\frac{C_{0}}{C_{I}} \times 100 \%
$$

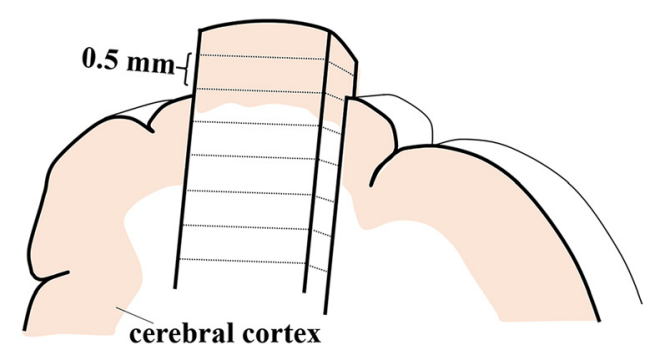

Figure 2: Drawing of a coronal section of one of the cerebral hemispheres. The strips of brain tissue used for analysis were cut from the coronal section. The parallel lines running across strip illustrate the subsequent serial sectioning of this strip into $0.5 \mathrm{~mm}$ thick slices. Figure modified from Levin et al. (1970). 
where $C_{O}$ is the equilibrium tissue concentration and $C_{I}$ is the inflow solution concentration. The experiment concluded that the ISS space in the larger animals, i.e., rabbit, cat, dog, and monkey, is about $17-20 \%$. In addition, the physical parameters of diffusion were measured, which are the effective diffusion coefficient $\left(D^{\star}\right)$ and the free diffusion coefficient (D), respectively (Levin et al. 1970). The technique was refined over the next few years. The value of $\mathrm{D}^{\star}$ was estimated by the following equation

$$
\frac{C_{x}}{C_{0}}=\operatorname{erfc}\left(\frac{x}{2 \sqrt{D * t}}\right)
$$

where erfc stands for the complementary error function. $C_{x}$ stands for concentration at distance $x$ from the ventricular border of the caudate nucleus (the detected brain region), where concentration is $C_{O}$ (Patlak and Fenstermacher 1975).

In the analysis of the brain ISS, the tortuosity $\lambda$ is a common calculation parameter that characterizes the hindrance to diffusion in the tested tissue compared to that in a free medium, which is calculated from the ratio of the two experimental measurements as:

$$
\lambda=\sqrt{D / D^{*}}
$$

The radiotracer data indicated a $\lambda$ between 1.4 and 1.7 (Blasberg et al. 1975; Fenstermacher and Kaye 1988; Fenstermacher et al.1974; Rosenberg et al. 1980). While the technique was effective, it relied on postmortem measurements of concentration in fixed and sectioned tissue and therefore did not provide spatial and dynamic information. These limitations have largely eliminated the use of the method.

Since the width of ISS is nanoscale, its ultrastructure should only be monitored by electron microscopy (EM), which is the only imaging method capable of providing a detailed structure of the nervous system. However, the traditional EM using chemical fixation showed a space of less than $5 \%$, which is due to the significant shrinkage and distortion of the tissue (Karlsson and Schultz 1964; Katzman 1961). During this stage, researchers had been trying to explore new fixation methods to overcome the failure of chemical fixation. Anton Van Harreveld introduced a method of rapidly freezing exposed living brains at a temperature of about $-207^{\circ} \mathrm{C}$, followed by substitution fixation in acetone containing 2 percent $\mathrm{OsO}_{4}$ at $-85^{\circ} \mathrm{C}$. Instantaneous freezing preserved the distribution of water. However, as the tissue slowly freezes, the formation of pure ice crystals condensed the solute. Freeze-substitution revealed a distinct extracellular space in the central nervous system (Vanharreveld et al. 1965). However, only the outer $20 \mu \mathrm{m}$ tissue is free from ice crystal damage, and the method is not widely used. The improvement of fixed methods has never stopped. Based on the viewpoint that the loss of ISS during traditional fixation was caused by the cell swelling to replace the ISS due to ingestion of water, Cragg attempted to perfuse the brain from the aorta with isotonic sucrose $(0.28 \mathrm{M})$ solution sequentially under highpressure $(300 \mathrm{~mm} \mathrm{Hg}$ ) and physiological pressure; this opened the blood-brain barrier and washing out the extracellular fluid with isotonic sucrose without changing the size of the cell. It was then fixed with a 1:1 mixture of isotonic solution of $1 \%$ glutaraldehyde with $1 \%$ formaldehyde in phosphate buffer and isotonic sucrose. This method succeeded in preserving ISS (Cragg 1979). Recently, Korogod and colleagues used rapid, highpressure freezing on adult mouse neocortex. The tissue was cut into approximately $200 \mu \mathrm{m}$ thick and was then high-pressure frozen. Subsequent procedures, including staining, dehydration, and embedding, were performed at a low temperature. The physical parameters of ISS were quantified by 3D reconstruction and measurement of the electron microscope images by using image analysis software, e.g., FIJI software. Compared with chemical fixation, cryogenic fixation not only retained a physiological extracellular space, but also revealed larger numbers of docked synaptic vesicles, a smaller glial volume, and a less intimate glial coverage of synapses and blood vessels (Figure 3) (Korogod et al. 2015).

\section{The establishment of the gold standard}

This period began with the emergence of the real-time detection of living tissue. Still, based on the biophysics of diffusion, the "point source paradigm" theory was applied to ISS exploration. The realization of this theory depends on the use of specific small monovalent ions and micropipettes or microelectrodes for both releasing ions and sensing the concentration of the ions. The probe ions were released in the form of a point source into the brain ISS by a constant current pulse to the glass micropipette with a tip diameter of a few micrometers and freely diffused away from the point source along random paths. Ion-selective microelectrodes (ISMs) positioned roughly $100 \mu \mathrm{m}$ away from the release point determined transient concentration changes of the probe ion. The voltage on the ISM represented the number of molecules, i.e., concentration (Nicholson 1993; Nicholson and Phillips 1981). From those measurements, $\alpha$ and the $\lambda$ can be calculated. The real-time iontophoresis (RTI) 
A

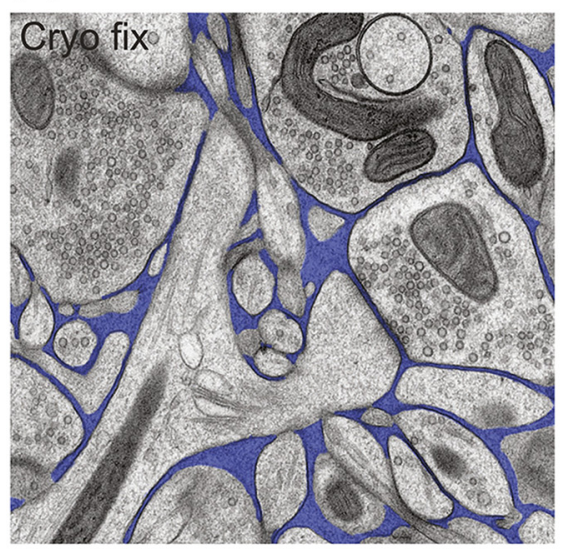

B

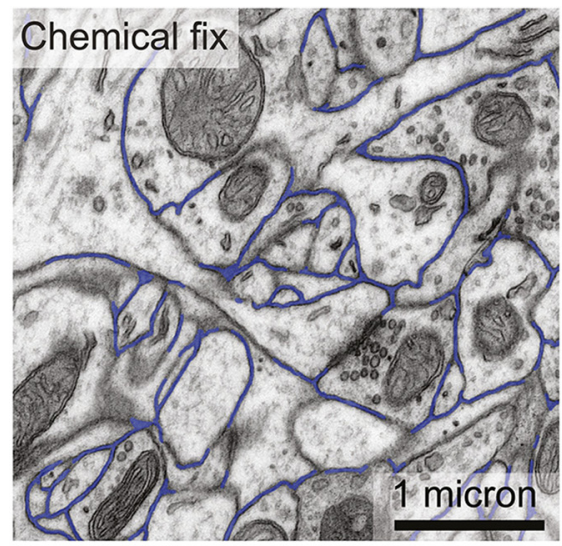

Figure 3: Chemical fixation reduces cortical volume and extracellular space. (A) The EM of cryofixed neuropil. (B) The EM of conventional chemically fixed neuropil. The volume fraction of ISS (pseudocolored in blue) decreases after chemical fixation. Figure modified from Korogod et al. (2015). method, based on the "point source paradigm" theory, had become the gold standard for quantifying the ISS of the brain, and been used extensively (Figure 4). The method can track any changes in the diffusion properties of brain tissue in both physiological and pathological conditions in real-time. The most commonly used exogenous probe ion for the RTI is the small monovalent cation tetramethylammonium (TMA) with $M_{r}=74$. Moreover, cation tetraethylammonium and the anions a-naphthalene sulfonate and hexafluoroarsenate have also been used (Nicholson and

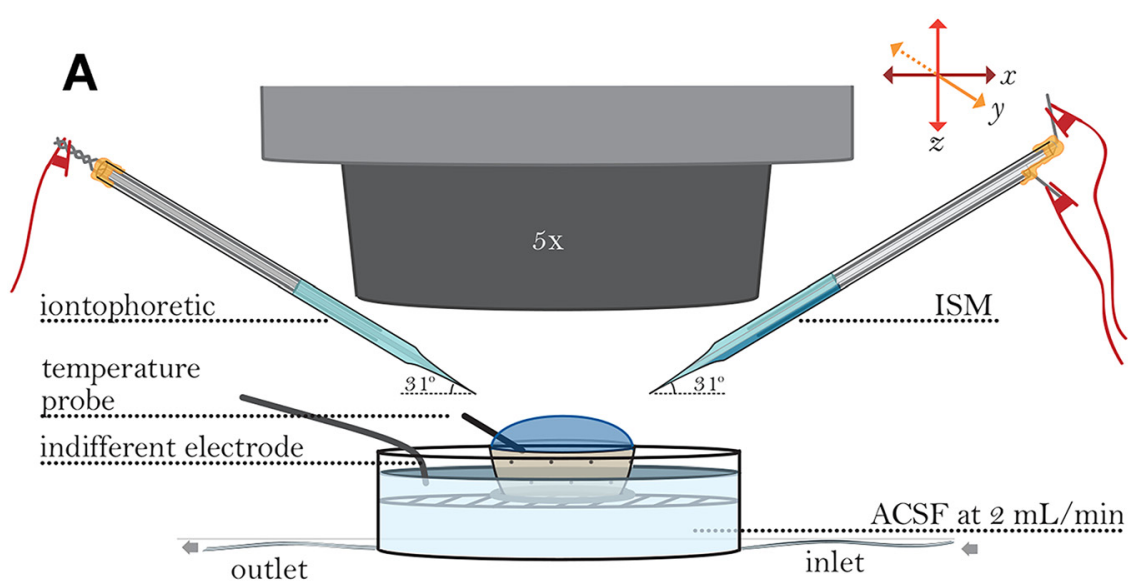

B

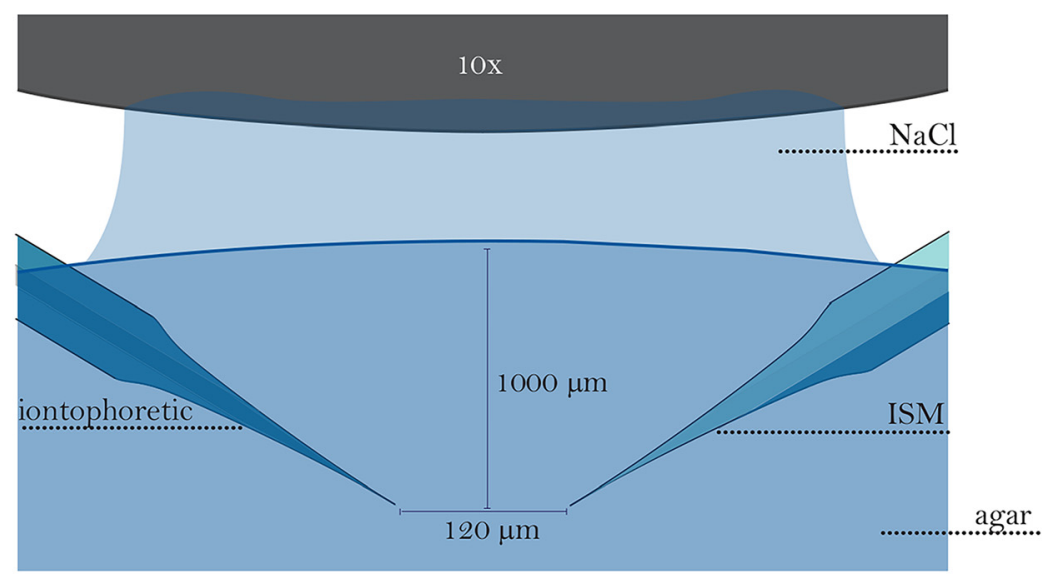

Figure 4: Setup for RTI experiments in agar. (A) Setup for the experiment in dilute agar. A small porous container containing diluted agar gel is placed in a flow perfusion chamber. An iontophoresis microelectrode (left side) and an ISM (right side) are held by microelectrode holders. A temperature probe is placed in the agar gel and an indifferent grounding electrode is placed in the immersion chamber. (B) Enlarged image of microelectrodes in agar gel. Microelectrodes are positioned to a depth of $1000 \mu \mathrm{m}$. The distance between the microelectrodes is $120 \mu \mathrm{m}$.

Figure modified from Odackal et al. (2017). 
Phillips 1981). The RTI-TMA data has determined that $\alpha$ is $\sim 0.2$ in most adult brain tissues, and the $\lambda$ is generally $\sim 1.6$, confirming the earlier research results.

Although the implementation of RTI is an outstanding advance in ISS research methods, there are several limitations. This method's utilization precondition is tissue homogeneity and isotropy. However, the cells in different brain regions are arranged in very distinct ways, which is why the distance between a point source and the ISM is not too large, usually roughly $80-130 \mu \mathrm{m}$. Moreover, when less than $50 \mu \mathrm{m}$, the ISM response may not be fast enough to record concentration changes in probe molecules (Odackal et al. 2017). Saghyan and colleagues, in their study of laminar brain structures exemplified by the hippocampus, derived a generalized diffusion equation for inhomogeneous environments and confined numerical solution for layered structures (Saghyan et al. 2012).

By this time, techniques were developed to precisely measure the diffusion characteristics of small molecules/ ions in local regions of the brain. However, many candidate molecules as extracellular signals, trophic factors, or protein-based drugs can be large, and the previous research methods were no longer applicable. Nicholson and colleagues developed an optical technique, known as integrative optical imaging (IOI), which enabled precise determination of the diffusion properties of fluorescently labeled macromolecules of different sizes and also revealed the ISS's typical width. Macromolecules labeled with a fluorophore were releases from a micropipette and then imaged by using a fluorescence microscope. The method was based on a re-evaluation of the theory of out-of-focus images without the need to optically section the tissue; therefore, the resulting image was not in the conventional sense, but a cloud of photons mapped onto the memory of a computer. The recorded image was fitted with a theoretical expression. From this fitting, the diffusion parameters were obtained (Hrabetova and Nicholson 2007; Nicholson and Tao 1993).

\section{Quantitative visualization of dynamics in the ISS}

Previous studies were unable to establish the true shape of the ISS. The advent of powerful imaging technologies is beginning to assist in its imaging, adding in the bibliography relevant to ISS exploration. This section will present three recent visualization approaches based on magnetic resonance imaging (MRI) (tracer-based MRI) and superresolution microscopy [single-molecule imaging of singlewalled carbon nanotubes (SWCNTs)] and super-resolution shadow imaging (SUSHI) that can visualize the ISS and extract information about its local viscosity, $\lambda$ or $\alpha$ in living brain tissue.

Han et al. investigated the ISS in vivo animal models using the tracer-based MRI technique which can not only globally visualize the dynamic transportation of tracers in the ISS of deep brain regions, but also quantifies diffusion parameters in tracer transportation and ISS structure. Gadolinium-diethylene triamine pentaacetic acid (Gd-DTPA), as a tracer, was stereotaxically injected into the ISS of the animal's target brain regions. Gd-DTPA is commonly used as a positive contrast agent to enhance MR image signal intensity (SI) (Ibrahim et al. 2020), which can be weakened due to the time-dependent diffusion and elimination of tracer in ISS (Figure 5). The change of SI is a linear function of the local Gd-DTPA concentration. Therefore, by measuring the SI shown on the MR image, the dynamic process involved in Gd-DTPA in ISS can be tracked in real-time, and the biophysical properties conform to the classical diffusion equation. The advantage of Gd-DTPA as a tracer of ISS lies in its biological inertness, thermal stability, and few translocations to the nerve cells (Caravan et al. 1999; Han et al. 2012). Compared with the difficult operation of RTI and the limited imaging thickness of IOI, tracer-based MRI is easier to achieve imaging of deeper tissues in vivo (Hou et al. 2017; Teng et al. 2018; Wang et al. 2019). It is beneficial for us to explore ISS in any brain region. A breakthrough was that ISS was proven to be a physiologically partitioned system that varies between different brain regions and, even within the same brain region, between different cortical laminae. Its division was characterized by the unique distribution territory and transportation ability of substances located in it, based on the experimental fact that although the caudate nucleus was adjacent to the thalamus, no tracer transport was observed between regions. Moreover, the effective diffusion coefficient $D^{\star}$ and $\lambda$ were statistically significant (Hou et al. 2017).

Super-resolution microscopy in living brain tissue broke new ground in studying brain ISS. It is believed that the single-molecule tracking technique should be suitable for revealing the ISS nanometric environment in living brain tissue. In single-molecule microscopy, the highly luminescent probes that can be efficiently individually detected by an optical microscope is usually used. SWCNTs, a near-infrared nanoprobe, were considered to be the most suitable candidate probes. SWCNTs have a higher spatial and temporal resolution and higher optical tissue penetration depth due to its long emission wavelength, less light scattering, and tissue spontaneous fluorescence interference. In 2009, Welsher and coworkers prepared a new type of SWCNTs by coating the surface with a layer of phospholipid-polyethylene glycol (PL-PEG) molecules to 


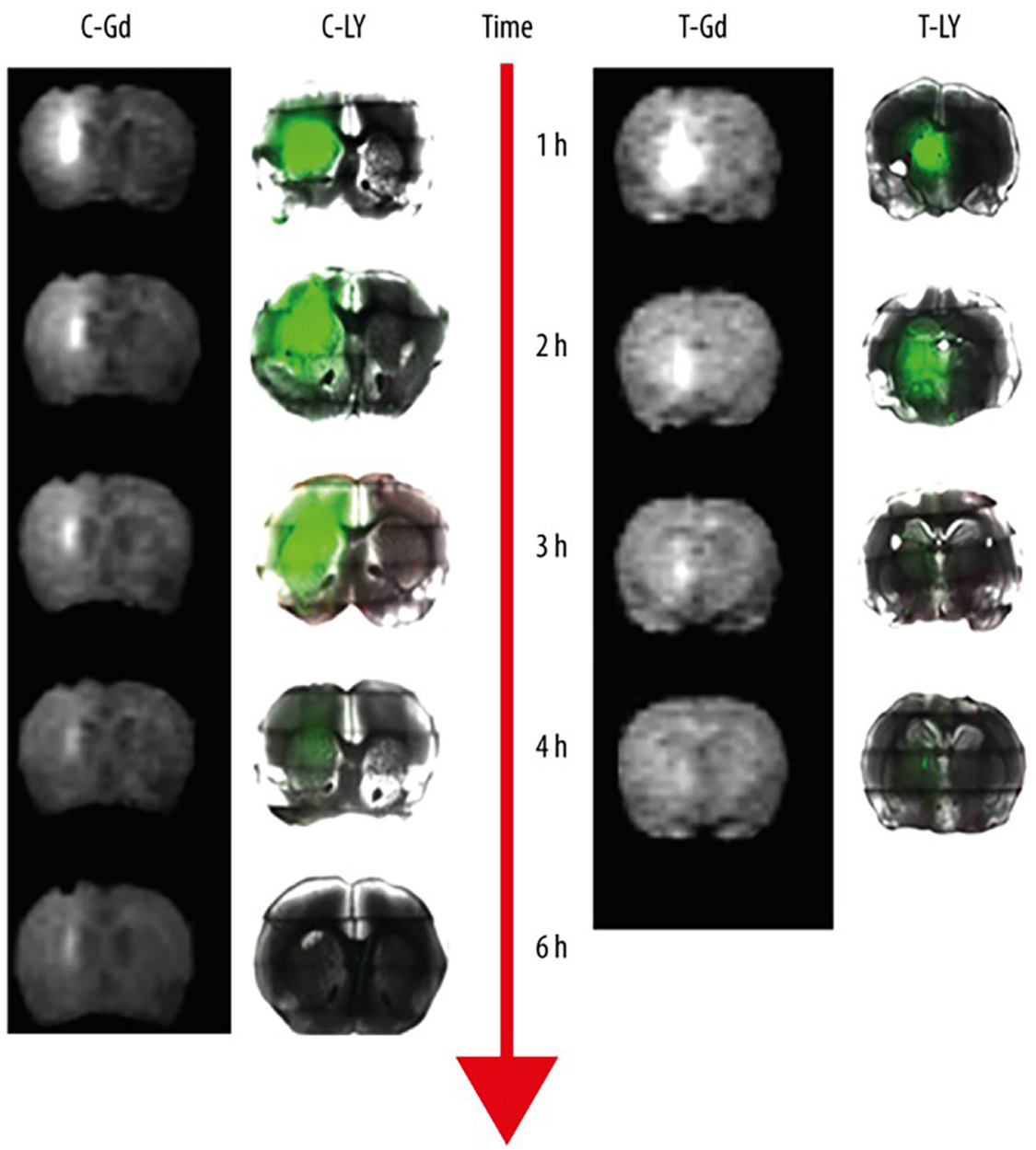

Figure 5: Transportation of Gd-DTPA and Lucifer Yellow in rat brain ISS. Gd-DTPA and Lucifer Yellow were injected into the caudate nucleus and transferred from the injection site to the ipsilateral frontal and temporal cortex, while both of them injected into the thalamus only stayed near the injection site. Although the caudate nucleus is close to the thalamus, Gd-DTPA and Lucifer Yellow did not transport between them. C-Gd, Gd-DTPA in the caudate nucleus; C-LY, Lucifer Yellow in the caudate nucleus; T-Gd, GdDTPA in the thalamus; T-LY, Lucifer Yellow in the thalamus. Figure modified from Hou et al. (2017).

improve their water solubility and biocompatibility and achieved the first whole animal imaging (Welsher et al. 2009). Moreover, the high aspect ratio and the rigidity of the SWCNTs with a length of 500-1000 $\mathrm{nm}$ and a diameter of $1-5 \mathrm{~nm}$ can moderate their diffusion rates at a fairly low level to be imaged at video rate for an extended period (Fakhri et al. 2010), which is almost impossible for spherical nanoparticles. Godin et al. combined super-resolution imaging with SWCNTs to directly observe the local ISS structures and rheology in brain tissue (Figure 6). PL-PEG
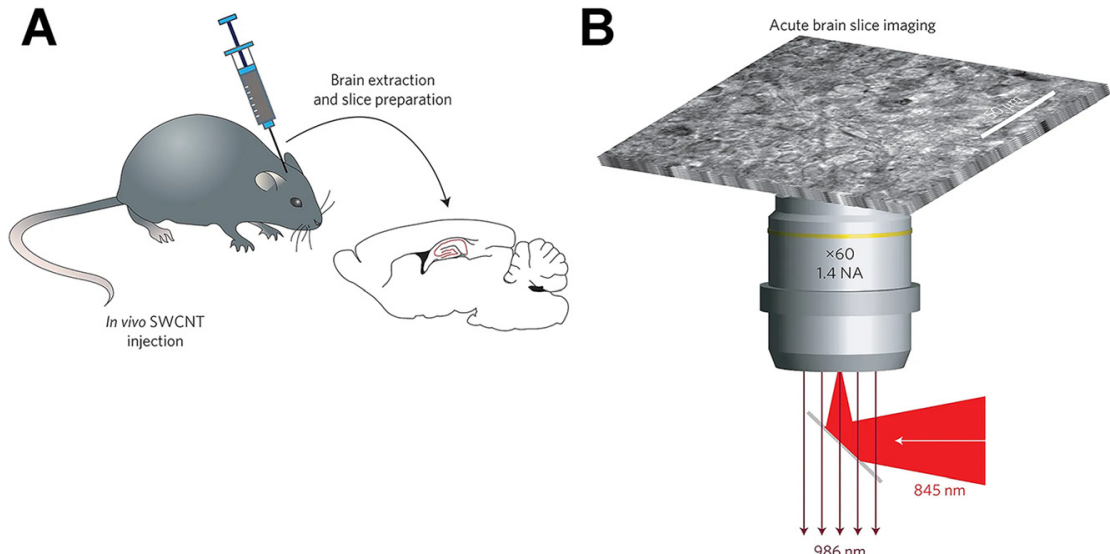

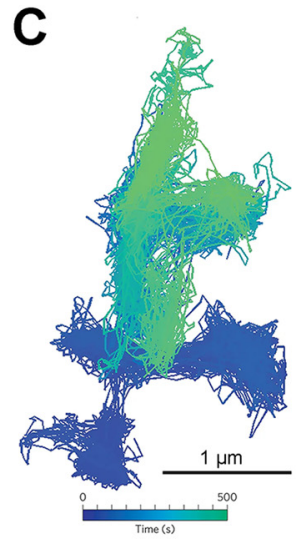

Figure 6: Single-molecule tracking of luminescent SWCNTs in ISS in living neonatal rat brain. (A) SWCNTs were injected into the lateral ventricles of living rats. (B) Luminescent SWCNTs were imaged in brain slices. (C) Color-coded trajectory of a single SWCNT diffusing in ISS in living cortical slice (20,000 data points). Figure modified from Godin et al. (2017). 
SWCNT solutions were delivered into lateral cerebroventricles of young rat brains. The snake slithering-like movements of individual SWCNT exploring the ISS could be resolved with video rate fluorescence imaging. The location of the nanotube center extracted from each film frame in the imaging plane was obtained by a twodimensional asymmetric Gaussian fitting analysis of the recorded long movies of a single SWCNT diffusion occurring over more than 20,000 frames. A large number of nanotube localization coordinates were pooled to create a super-resolution image of the ISS that directly unveiled the spatial, nanoscale, and structural $\lambda$ of acute brain slices which were very similar with those obtained by cryo-EM (Korogod et al. 2015; Van Harreveld and Steiner 1970). The SWCNT super-resolution imaging was also used to reveal a diverse ECS in both healthy and neurodegenerative context. Quantitative analysis detected that SWCNTs exploration covered a significantly broader ECS area in the brain of synucleinopathy mice (Soria et al. 2020). In sum, this optical approach revealed that the ISS is a maze of interconnected polymorphic compartments that are structured down to the nanoscale and bear specific diffusive properties (Godin et al. 2017).

The research by Tønnesen and colleagues yielded surprising outcomes. They combined three dimensionalstimulated emission depletion (3D-STED) microscopy and fluorescent labeling of the extracellular fluid with a diffusible fluorophore to develop SUSHI of brain ISS. SUSHI enables quantitative analysis of ISS spatial topology revealing dynamics. STED microscopy is a volumetric super-resolution imaging technique. In the standard STED approach, by silencing the peripheral fluorophores of the excited spot, leaving only the very center zone vigorous for fluorescence, the effective point spread function could be immensely squeezed, thus greatly improving the resolution (Li et al. 2018). This technique has been successfully applied to the real-time analysis of nanoscale neural morphology of living tissue. The 3D-STED can improve the spatial resolution in the $z$-direction by distributing the STED light intensity more isotropically around the excitation focus (Lenz et al. 2014; Wildanger et al. 2009). In SUSHI, the extracellular fluid was labeled with a diffusible biology fluorescent dyes, such as calcein and Alexa Fluor 488 , which stayed outside of the cells. The labeling strategy enabled visualization of the ISS, and the cell bodies and their processes appeared to be dark voids resembling shadows embedded in the surrounding fluorescent. Darkness and brightness were exchanged through the image's inverted grayscale lookup table. That is, cellular structures were inversely labeled and stand out, enabling detailed 3D reconstructions of unlabeled cells (Figure 7). Based on the high-contrast images provided by SUSHI, the authors estimated the ISS $\alpha$ by binarizing the images. $\alpha$ was then simply the ratio of the number of above-threshold pixels to the total number of pixels for a given region of interest, ranging from 5 to $36 \%$, with a mean of $19 \pm 10 \%$ in organotypic brain slices, and showing wide regional variations across different layers of the hippocampus and a relatively spacious distribution of the extracellular space around dendritic spines (Arizono et al. 2020). The width of the ISS was also quantified by analyzing intensity peaks in line profiles measured across the neuropil. Their widths ranged from around $50 \mathrm{~nm}$ to well above $1 \mu \mathrm{m}$ (Tonnesen et al. 2018). With SUSHI imaging, the synaptic clefts can be identified and the gap between pre- and postsynaptic contacts can be measured (Inavalli et al. 2019).

So far, although SUSHI provided unprecedented optical access to the structure and dynamics of the ISS, they were still only applied to brain slices. In theory, SUSHI is feasible in vivo using cranial windows, but craniectomy reduces arterial pulsatility and decreases glymphatic flow (Plog et al. 2019), which can cause some distortion in fluid dynamics and likely in drug distribution.

\section{Physiological function and pathological dysfunction of ISS}

\section{Physiological function}

ISS provides an essential microenvironment for the health of cells and brain homeostasis, which plays an important functional role in brain health and disease. The development of ISS studying methods has enabled the exploration of its functions. ECM is an important component of the ISS. The adult brain has a unique ECM composition with almost negligible presence of collagen and other fibrillar ECM proteins (Zimmermann and Dours-Zimmermann 2008). The ECM of the brain is enriched with proteoglycans, glycoproteins, small linker proteins, matricellular proteins, and important enzymes that regulate the ECM deposition and degradation. A large portion of the proteoglycans in the central nervous system (CNS) consists of chondroitin sulfate proteoglycans, including aggrecan, versican, neurocan, and brevican, collectively termed lecticans (Yamaguchi 2000). They are also known as hyalectans for their ability to bind to hyaluronic acid (HA; hyaluronan) (Iozzo 1998; Krishnaswamy et al. 2019), which are instrumental regarding the maintenance of ISS volume and normal brain activity (Perkins et al. 2017). A lack of HA leads to a reduction in the volume of ISS in the brain, which in turn either triggers or intensifies epileptic 
A

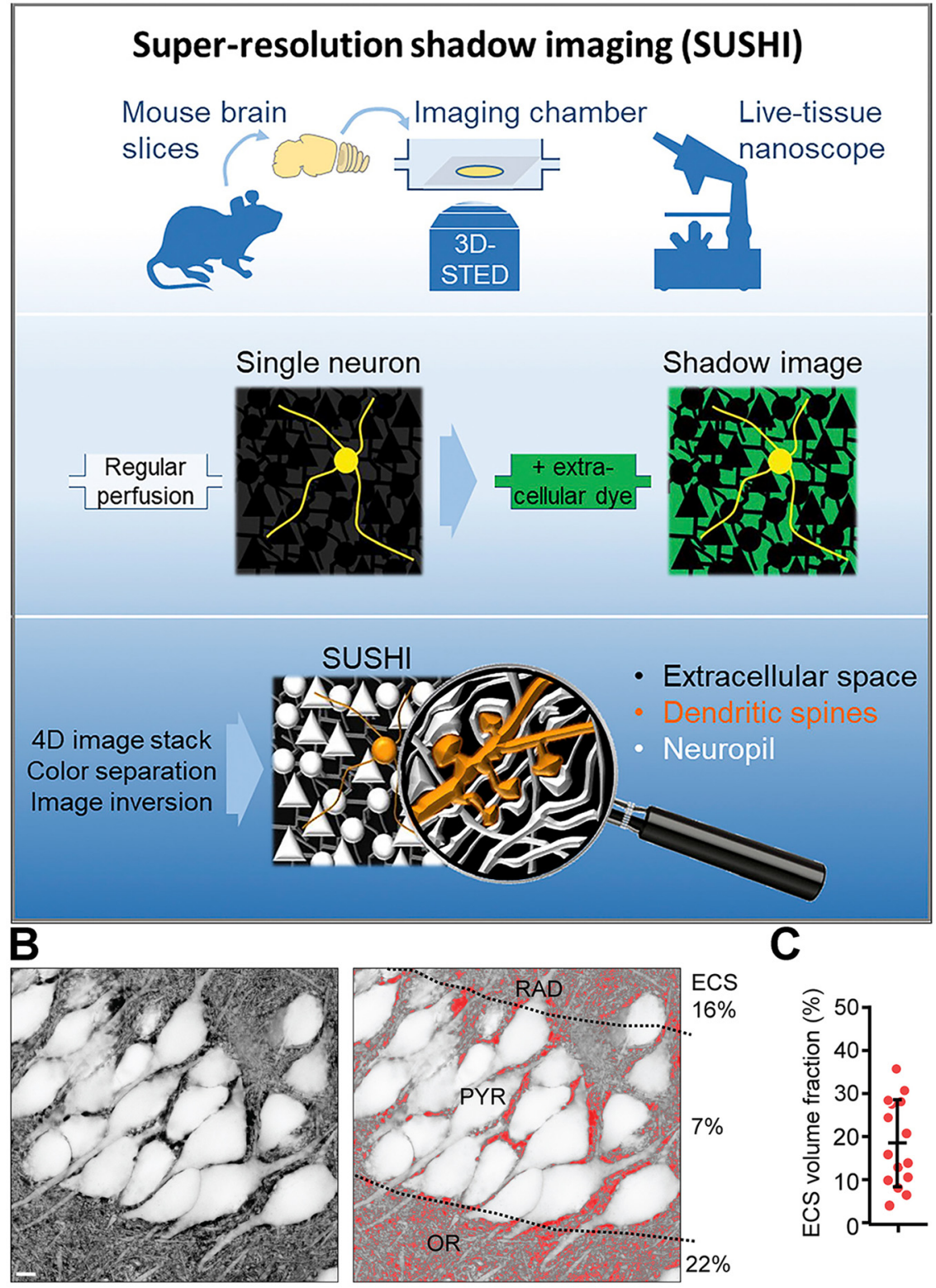

Figure 7: Super-resolution shadow imaging (SUSHI) of the ISS in living brain tissue. (A) Super-resolution 3D-STED microscopy combined with fluorescence labeling of the interstitial fluid reveals the complex spatial structure of the ISS in living brain tissue. (B) The overview of the hippocampal CA1 area, illustrating the different ISS volume fractions in the oriens (OR), stratum pyramidale (PYR), and stratum radiatum (RAD). (C) Statistical analysis of hippocampal volume fraction. Figure modified from Tonnesen et al. (2018). activity (Arranz et al. 2014). Its geometry constitutes a corridor for diffusion-mediated transport and clearance of substances, covering both relatively short distances (tens to hundreds of nanometers), such as chemical synapses and extrasynaptic receptors, and significantly longer distances (micrometers to centimeters), just like volume transmission and metabolite removal, where bulk flow may play a role (Hrabetova et al. 2018).

Compared to adults, ISS volume significantly increases during postnatal development, while $\lambda$ significantly decreases. Additionally, the effective diffusion coefficient in the first days of postnatal development was significantly lower than that of adults, and the clearance rate constant $\left(k^{\prime}\right)$ was greater than that of adults (Yang et al. 2016). Larger ISS channels during development facilitate the transport of large molecules, such as growth factors, providing better conditions for cell migration (Sykova 2004).

ISS stores all of the extracellular ions that are necessary to maintain the resting and action potential of cells and to allow the release of transmitter molecules on synapses. Compared with other ions, $\mathrm{K}^{+}$is a relatively more noteworthy signaling agent in the ISS. Fluctuations in local $\mathrm{K}^{+}$concentration are more likely to cause changes in membrane potential as extracellular $\mathrm{Na}^{+}$and $\mathrm{Cl}^{-}$concentrations are too high to be effectively regulated. During the neuronal action potential, the release of $\mathrm{K}^{+}$into the 
restrictive ISS results in transient increases in $\left[\mathrm{K}^{+}\right]_{\mathrm{o}}$ and associated glial cell swelling, which further induces the ISS shrinkage (Ballanyi et al. 1987). ISS and glial cells act as a buffer system for $\mathrm{K}^{+}$during neuronal activity.

In addition to the involvement of ion channel transport and intracellular signaling pathways in the homeostatic plasticity of neural networks, the importance of ECM molecules cannot be ignored. Emerging evidence suggests a synergistic interplay between ECM and metabotropic receptors in homeostatic plasticity. Several studies have revealed that the interaction between metabotropic glutamate receptors (mGluRs) and cell adhesion molecules (CAMs) plays a crucial role in regulating the localization and signaling of the mGluRs. ELFN1 [extracellular leucine-rich repeat (LRR) and fibronectin type III domain-containing 1] which is a member of the family of LRR CAMs, interacts trans-synaptically with class III mGluRs (Stachniak et al. 2019; Wang et al. 2017), thereby retaining mGluRs selectively at synapses between certain neurons. The enrichment of mGluRs reduces neurotransmitter release probability and endow these synapses with their distinctive short-term facilitation properties by negative feedback mechanisms (Tomioka et al. 2014). Not only can the extracellular environment control mGluRs, but the signal transduction of mGluRs can also regulate the extracellular environment. Stimulation of mGluRs activates the tumor necrosis factor- $\alpha$-converting enzyme (TACE). TACE-induced extracellular proteolysis involves in downregulation of excitatory transmission (Cho et al. 2008). Dopaminergic and serotonergic neuromodulation control the activity of disintegrin and metalloprotease with thrombospondin motifs (ADAMTS). ADAMTS-mediated proteolytic modifications of ECM are associated with inactivity-induced homeostatic synaptic upscaling (Valenzuela et al. 2014).

The transport through the ISS in the brain is involved in the "glymphatic" flow of cerebrospinal fluid from the paraarterial to the para-venous spaces, known as perivascular space (PVS) defined as the space between the penetrating arteries of the brain and the layer of pia mater that is carried inward with the developing vessels. The brain lacks the traditional lymphatic drainage system. Instead, glial cells act as a major part of the lymphatic pathway, known as the "glymphatic" pathway. Glymphatic transport occurs extensively in brain physiological and pathological processes, including clearance of proteins ( $\beta$-amyloid) linked to neurodegenerative diseases (Iliff et al. 2012) and toxic metabolites, and removal of excess fluid brain edema. Over short distances, most substances move through the narrow and tortuous ISS in brain parenchyma by a diffusive mechanism. Three kilodalton dextran molecules injected into the cisterna magna moved into the perivascular spaces around brain capillaries and then spread through the ISS before apparently leaving the brain via deep perivenous pathways. According to this result, it is believed that a bulk flow containing macromolecules performs this transport rather than diffusion. It was therefore proposed that the paravascular glymphatic pathway driven by bulk flow constitutes a major clearance pathway of interstitial fluid solutes from the brain's parenchyma (Iliff and Nedergaard 2013). But the glymphatic mechanism and role of aquaporin-4 (AQP4) has been questioned (Hladky and Barrand 2014; Spector et al. 2015). The efficiency of glymphatic transport increases significantly during sleep and is associated with an increase in $\alpha$ in the cortex, suggesting that this was a mechanism for removing metabolic waste from the brain (Xie et al. 2013). Furthermore, the sleeping brain shows cerebrospinal fluid (CSF) flow on a macroscopic scale, which is expected to alter waste removal because pulsating fluid dynamics can increase mixing and diffusion (Fultz et al. 2019).

\section{Pathological dysfunction}

Alterations in the physical and physiological parameters of ISS, including $\alpha, \lambda$, and extracellular matrix components have been linked to brain development, aging, trauma, and many other fundamental processes and diseases of the brain. The changes in these parameters may serve as important indicators of pathological processes and posttreatment recovery.

Edema under various pathological conditions, usually accompanied by the swelling of astrocytes, is believed to be the major cause of changes in the ISS diffusion parameters. Recent studies suggest that neurons also contribute to edema formation (Murphy et al. 2017; Rungta et al. 2015). The used models of cytotoxic edema included: anoxia, oxygen-glucose deprivation, ischemia, hypotonic stress, and hypoxia (Chmelova et al. 2019). Both neuron and glia swelling during hypoxia and energy substrate deprivation are caused by large ionic shifts in the ISS. $\left[\mathrm{K}^{+}\right]_{\mathrm{e}}$, extracellular acidification, and excitatory amino acids, particularly glutamate, significantly increase, together with an intracellular $\mathrm{Na}^{+}$and $\mathrm{Cl}^{-}$accumulation (Hansen 1985; Sykova 1983, 2004). Transmembrane ion transfer leads to an osmotically forced water influx and the development of cytotoxic edema, profoundly impacting brain cell function. Due to the limitations of the skull, the cell swelling inevitably leads to a reduction in ISS volume, followed by the decrease of $\alpha$ from about 0.20 to about 0.05 and the increase of the $\lambda$ from 1.5 to 2.1 (Lundbaek and Hansen 1992; Sykova et al. 1994). After an ischemic stroke, CSF flowed rapidly into brain tissue along perivascular spaces, 
causing swelling (Mestre et al. 2020). The swelling after stroke leads to an increase in the area of dead space in ISS, block the drainage pathway of ISF, and eventually delay the diffusion (Arbel-Ornath et al. 2013; Zoremba et al. 2007).

Due to the biophysical changes in the ISS, the impaired ability of substance transportation has contributed to a series of brain diseases, especially neurodegenerative. It is plausible that either the causality is reversed or the two are mutually reinforcing. Research on ISS diffusion parameters of neurodegenerative diseases mainly focuses on Alzheimer's and Parkinson's disease ( $\mathrm{AD}$ and $\mathrm{PD}$, respectively). A core feature of $\mathrm{AD}$ is the increase in amyloid- $\beta(A \beta)$ peptide synthesis by neurons and aggregates of $A \beta$ which form plaques in the ISS, which worsens with advancing age. AD model mice exhibited a marked decrease in ISF flow and an increase in ISS volume (Sykova et al. 2005). There is evidence to support that $A \beta$ deposition in the ISS directly blocked ISF flow from the superficial cortex-caudate nucleus into the deep brain to the third ventricle and then disturbed the ISF-CSF cycle between the cortex and hippocampus in AD mice. This suggests that $A \beta$-blocked ISF flow contributes to the onset of AD (Yue et al. 2019). Brain ISS disturbances have been implicated in PD pathogenesis. The MRI tracerbased method demonstrated the change of ISS diffusion parameters in a PD model, including a decrease in $\lambda$ and an increase in $D^{*}$ in the 6-OHDA-lesioned substantia nigra; however, $k^{\prime}$ was inconsistent (Fang et al. 2017; Ren et al. 2016). PD is accompanied by astrogliosis and changes in the extracellular matrix, which in principle can cause the increase in $\lambda$ and the decrease in $k^{\prime}$ (Roitbak and Sykova 1999; Sykova et al. 1998). A possible explanation is that dopamine neurons are approximately five times longer than astrocytes; thus, the progressive loss of more than $75 \%$ of the neurons in the SN was the primary contributor to the reduction in $\lambda$ and an increase in $D^{*}$ (Reum et al. 2002).

The growth of brain tumors, especially gliomas, can destroy the normal structure of the brain tissue. The ISS volume fraction has therefore been recognized as a strong biomarker for tumor response and treatment efficacy (Jain 1987). Cell migration may depend largely on the size and geometry of the ISS. Compared to the average values of $\alpha$ (0.24) and $\lambda$ (1.55) in normal brain tissue, the average $\alpha$ was as large as $\sim 50 \%$ in anaplastic astrocytomas and glioblastomas, and the increase in $\lambda$ to $1.67-1.77$ can be attributed to astrogliosis (Han et al. 2014; Li et al. 2013; Reynaud et al. 2016; Vargova et al. 2003; Zamecnik et al. 2004). The lower $D^{*}$ and $k^{\prime}$, and a longer half-life were also found in gliomas. The mechanisms behind the altered diffusion parameters are associated with distinct glioma ECM components. ECM components such as chondroitin sulfate proteoglycan, tenascin C, and type IV collagen were elevated in glioma (Song et al. 2017).

\section{Detailed knowledge of the ISS network foster new strategies for drug delivery}

New CNS medications have been suffering from the poorest success rates compared to other therapeutic areas. The main challenge is that numerous lipid-insoluble small molecules and most macromolecules drugs are largely unable to achieve sustained and targeted CNS delivery. The greatest hurdle for effective CNS delivery is the blood-brain barrier (BBB), which limits the transfer of substances between the plasma and the interstitial of the CNS. Various new drug delivery strategies based on overcoming the BBB have been explored, including drug delivery carriers (e.g., viral vectors, nanoparticles, and exosomes), active transporters, brain permeability enhancers (e.g., mannitol, and puerarin with borneol), non-invasive techniques (e.g., microbubbleenhanced diagnostic ultrasound), and alteration of administration routes (e.g., intranasal delivery) (Dong 2018). Direct intracranial delivery would eliminate the need for drugs to cross the BBB. The discovery of a subdivision drainage system in the brain opens up the possibility of ISS as a new approach to drug delivery, which can succeed in bypassing the BBB that prevents most drugs from reaching the brain. Most drugs that have failed to be developed orally or intravenously can be revived. This review describes three of the promising approaches for direct intracranial delivery of agents: polymeric-controlled release, convection-enhanced delivery, and simple diffusion delivery.

\section{Polymeric-controlled release system}

The polymeric-controlled release provides a way for local delivery of tumor chemotherapy to circumvent the BBB, allowing delivery of chemotherapy drugs directly to malignant cells in the brain. Controlled release systems are designed from both degradable and nondegradable polymers as carriers of chemotherapy, which are implanted at the time of tumor removal or biopsy, and therefore not requiring additional invasive procedures (Saini et al. 2004) (Figure 8). Polymers introduce a sustained drug-source for up to months after one single treatment and maximize local concentration while minimizing systemic toxicity. 


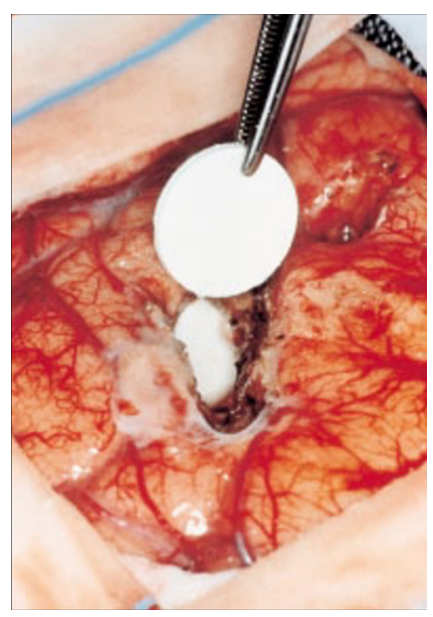

Figure 8: Intracranial placement of Gliadel ${ }^{\circledR}$ wafer. The wafer is implanted into a human brain following surgical resection of the tumor. Each wafer is $14 \mathrm{~mm}$ in diameter, $1 \mathrm{~mm}$ in thickness. Figure modified from Fleming and Saltzman (2002).

Biodegradable polymers are more common as controlledrelease carriers than nondegradable systems. The common copolymer systems used intracranially include polybis (p-carboxyphenoxy) propane-sebacic acid (DiMeco et al. 2002; Hsu et al. 2005), fatty acid dimer (Lin et al. 2005), polylactic acid, polyglycolic acid, and their copolymer polylactic-co-glycolic acid (PLGA) (Mu and Feng 2003). More recently, $N$-(2-hydroxypropyl) methacrylamide (HPMA) copolymer biomaterials, a water-soluble synthetic polymer carrier is studied in-depth, which has excellent water solubility, biocompatibility, and non-immunogenic properties. The high molecular-weight star-like polymeric materials based on HPMA copolymers show superior efficacy and tumor accumulation (Kostka et al. 2020). The diffusion distance of the controlled-release system and local drug concentration is limited by the diffusion characteristics of ISS. Low diffusion coefficients and a high rate of elimination result in drug delivery distance limited to millimeters (Haller and Saltzman 1998). Therefore, considering poor drug penetration and limited drug dosage by the implant size, one of the research directions of polymer drug delivery systems should increase the size or be used in enough amount to deliver clinically relevant drug dosage (Sawyer et al. 2006).

\section{Convection-enhanced delivery}

Convection-enhanced delivery (CED) makes up for the defects of polymeric-controlled release systems to some extent. CED was first proposed and introduced by Bobo and coworkers in 1994 (Bobo et al. 1994) to facilitate the delivery of drugs that are too large to effectively diffuse over required distances and bypass BBB. In CED, an infusion catheter is connected to a syringe pump, which maintains the desired pressure gradient, to deliver therapeutics directly through the interstitial spaces of the brain (Figure 9). It can achieve an infusion distance of more than $1 \mathrm{~cm}$ from the site of injection and large volumes of high drug concentrations with minimum systemic toxicity (Garg et al. 2015). Polymeric nanocarrier for the delivery of medications by CED can further improve the antitumor efficacy. The PLGA nanoparticle vector (Arshad et al. 2015), polyethylene glycol dimethacrylate injectable hydrogel (Fourniols et al. 2015), and liposomes (NordlingDavid et al. 2017; Shi et al. 2018) demonstrated various antitumor degrees, increased distribution, reduced clearance, and decreased neuronal toxicity. The main challenge for CED is to achieve an optimal infusion rate to avoid backflow along the cannula track. In principle, it is considered the optimal infusion rate when the therapeutic volume is delivered in the least amount of time without any reflux (Garg et al. 2015). CED has been a powerful tool for drug delivery used to deliver a variety of therapeutic compounds (neurotrophic factors, antibodies, growth factors, genetic vectors, and enzymes) for a diversity of neurodegenerative diseases, such as Alzheimer's disease and Parkinson's disease, and glioblastoma tumors.

\section{Simple diffusion delivery}

Han et al. (2011) proposed a novel technique for delivering drugs into the brain, named the simple diffusion delivery

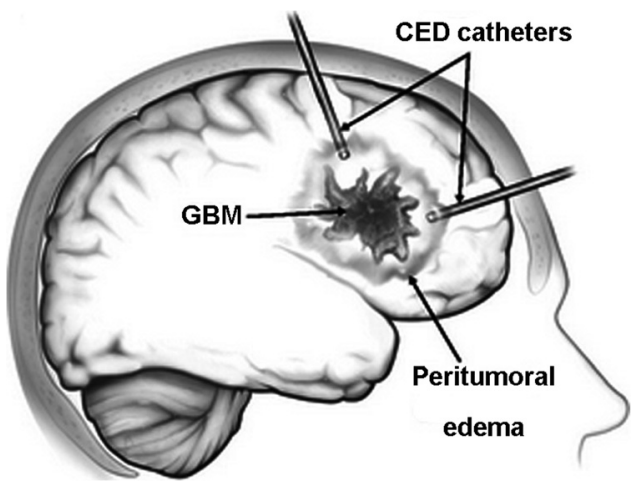

Figure 9: Process and characteristics of convection-enhanced delivery. One or more catheters are inserted into the interstitial spaces of the brain. A consistent, pressure-gradient, generated using an infusion pump, deliver therapeutic agents homogeneously into clinically significant volumes of distribution. CED: convectionenhanced delivery; GBM: glioblastoma multiforme. Figure modified from Reardon et al. (2006). 
(SDD). It is based on the findings of the brain ISS divisions and the properties of substance transport in each division by tracer-based MRI technique. SDD has shown outstanding advantages in the treatment of stroke models, based on the fact that in the absence of recanalization of blood vessels following ischemia, the traditional administration methods, such as oral and intravenous administration, provide lower volumes of drugs into brain tissue. The upstream of the ISF drainage area in the ISS partition of the middle cerebral artery blood supply zone was used as a drug import point, where cytidine diphosphate choline (CDPC) was stereotaxically delivered. Drug molecules can travel to brain regions by diffusion without the aid of external pressure. According to the diffusion rate of water-soluble small molecules, the distribution of drug molecules in ISS and the time and concentration range of reaching the proposed protection zone were estimated, and the prophylactic administration was finally determined. Only 1/800 conventional dose of CDPC achieved six times the neuroprotective effect, better than the conventional route (Xu et al. 2011). Compared with CED, the SDD process without continuous pressure avoids unwanted backflow, uncontrollable distribution, and tissue damage.

\section{Summary and future prospects}

With the deepening of understanding of the brain ISS, the study of the CNS is expected to upgrade from two dimensions only considering brain cells and blood vessels to three. The new research model that takes into account brain cells, cerebral vessels, and ISS will provide a new perspective and direction for understanding, utilizing, and protecting the brain (Lei et al. 2017). From the biophysical perspective, the diffusion properties of ISS can be used both to detect its elusive structure and to reveal the major mechanisms for the transportation of molecular signals and neuromodulators (Nicholson and Hrabetova 2017). From the perspective of drug delivery strategies, ISS will need to be further developed as an important conduit to bypass the BBB for intracranially local drug delivery. On the contrary, its diffusion properties also affect the distribution and clearance of drug molecules, so changing the diffusion properties may improve the therapeutic effect of medication.

Author contributions: All the authors have accepted responsibility for the entire content of this submitted manuscript and approved submission.

Research funding: This review was financially supported by the National Natural Science Foundation of China (no. 81401259) and Science and Technology Major Projects: Significant New-Drugs Creation (Grant no 2017ZX09101003-009-006).

Conflict of interest statement: The authors declare no conflict of interest, financial or otherwise.

\section{References}

Allen, J.N. (1955). Extracellular space in the central nervous system. AMA Arch. Neurol. Psychiatr. 73: 241-248.

Arbel-Ornath, M., Hudry, E., Eikermann-Haerter, K., Hou, S., Gregory, J.L., Zhao, L., Betensky, R.A., Frosch, M.P., Greenberg, S.M., Bacskai, B.J. (2013). Interstitial fluid drainage is impaired in ischemic stroke and Alzheimer's disease mouse models. Acta Neuropathol. 126: 353-364.

Arizono, M., Inavalli, V., Panatier, A., Pfeiffer, T., Angibaud, J., Levet, F., Ter Veer, M.J.T., Stobart, J., Bellocchio, L., Mikoshiba, K., et al. (2020). Structural basis of astrocytic $\mathrm{Ca}^{2+}$ signals at tripartite synapses. Nat. Commun. 11: 1906.

Arranz, A.M., Perkins, K.L., Irie, F., Lewis, D.P., Hrabe, J., Xiao, F., Itano, N., Kimata, K., Hrabetova, S., Yamaguchi, Y. (2014). Hyaluronan deficiency due to Has3 knock-out causes altered neuronal activity and seizures via reduction in brain extracellular space. J. Neurosci. 34: 6164-6176.

Arshad, A., Yang, B., Bienemann, A.S., Barua, N.U., Wyatt, M.J., Woolley, M., Johnson, D.E., Edler, K.J., Gill, S.S. (2015). Convection-enhanced delivery of carboplatin PLGA nanoparticles for the treatment of glioblastoma. PloS One 10: e0132266.

Ballanyi, K., Grafe, P., Ten Bruggencate, G. (1987). Ion activities and potassium uptake mechanisms of glial cells in Guinea-pig olfactory cortex slices. J. Physiol. 382: 159-174.

Blasberg, R.G., Patlak, C., Fenstermacher, J.D. (1975). Intrathecal chemotherapy: brain tissue profiles after ventriculocisternal perfusion. J. Pharmacol. Exp. Therapeut. 195: 73-83.

Bobo, R.H., Laske, D.W., Akbasak, A., Morrison, P.F., Dedrick, R.L., Oldfield, E.H. (1994). Convection-enhanced delivery of macromolecules in the brain. Proc. Natl. Acad. Sci. USA. 91: 2076-2080.

Caravan, P., Ellison, J.J., McMurry, T.J., Lauffer, R.B. (1999). Gadolinium(III) chelates as MRI contrast agents: structure, dynamics, and applications. Chem. Rev. 99: 2293-2352.

Chmelova, M., Sucha, P., Bochin, M., Vorisek, I., Pivonkova, H., Hermanova, Z., Anderova, M., Vargova, L. (2019). The role of aquaporin-4 and transient receptor potential vaniloid isoform 4 channels in the development of cytotoxic edema and associated extracellular diffusion parameter changes. Eur. J. Neurosci. 50: 1685-1699.

Cho, R.W., Park, J.M., Wolff, S.B., Xu, D., Hopf, C., Kim, J.A., Reddy, R.C., Petralia, R.S., Perin, M.S., Linden, D.J., et al. (2008). mGluR1/ 5-dependent long-term depression requires the regulated ectodomain cleavage of neuronal pentraxin NPR by TACE. Neuron 57: 858-871.

Cragg, B. (1979). Brain extracellular space fixed for electron microscopy. Neurosci. Lett. 15: 301-306.

DiMeco, F., Li, K.W., Tyler, B.M., Wolf, A.S., Brem, H., Olivi, A. (2002). Local delivery of mitoxantrone for the treatment of malignant brain tumors in rats. J. Neurosurg. 97: 1173-1178. 
Ding, F., O’Donnell, J., Xu, Q., Kang, N., Goldman, N., Nedergaard, M. (2016). Changes in the composition of brain interstitial ions control the sleep-wake cycle. Science 352: 550-555.

Dong, X. (2018). Current strategies for brain drug delivery. Theranostics 8: 1481-1493.

Fakhri, N., MacKintosh, F.C., Lounis, B., Cognet, L., Pasquali, M. (2010). Brownian motion of stiff filaments in a crowded environment. Science 330: 1804-1807.

Fang, Y., Dong, Y., Zheng, T., Du, D., Wen, J., Gao, D., Liu, L. (2017). Altered tracer distribution and clearance in the extracellular space of the substantia nigra in a rodent model of Parkinson's disease. Front. Neurosci. 11: 409.

Fenstermacher, J. and Kaye, T. (1988). Drug "diffusion" within the brain. Ann. N. Y. Acad. Sci. 531: 29-39.

Fenstermacher, J.D., Patlak, C.S., Blasberg, R.G. (1974). Transport of material between brain extracellular fluid, brain cells and blood. Fed. Proc. 33: 2070-2074.

Fleming, A.B. and Saltzman, W.M. (2002). Pharmacokinetics of the carmustine implant. Clin. Pharmacokinet. 41: 403-419.

Fourniols, T., Randolph, L.D., Staub, A., Vanvarenberg, K., Leprince, J.G., Preat, V., Des Rieux, A., Danhier, F. (2015). Temozolomide-loaded photopolymerizable PEG-DMA-based hydrogel for the treatment of glioblastoma. J. Contr. Release 210: 95-104.

Fultz, N.E., Bonmassar, G., Setsompop, K., Stickgold, R.A., Rosen, B.R., Polimeni, J.R., Lewis, L.D. (2019). Coupled electrophysiological, hemodynamic, and cerebrospinal fluid oscillations in human sleep. Science 366: 628-631.

Garg, T., Bhandari, S., Rath, G., Goyal, A.K. (2015). Current strategies for targeted delivery of bio-active drug molecules in the treatment of brain tumor. J. Drug Target. 23: 865-887.

Godin, A.G., Varela, J.A., Gao, Z., Danne, N., Dupuis, J.P., Lounis, B., Groc, L., Cognet, L. (2017). Single-nanotube tracking reveals the nanoscale organization of the extracellular space in the live brain. Nat. Nanotechnol. 12: 238-243.

Haller, M.F. and Saltzman, W.M. (1998). Localized delivery of proteins in the brain: can transport be customized? Pharmaceut. Res. 15: 377-385.

Han, H., Li, K., Yan, J., Zhu, K., Fu, Y. (2012). An in vivo study with an MRI tracer method reveals the biophysical properties of interstitial fluid in the rat brain. Sci. China Life Sci. 55: 782-787.

Han, H., Shi, C., Fu, Y., Zuo, L., Lee, K., He, Q., Han, H. (2014). A novel MRI tracer-based method for measuring water diffusion in the extracellular space of the rat brain. IEEE J. Biomed. Health Inf. 18: 978-983.

Han, H., Xia, Z., Chen, H., Hou, C., Li, W. (2011). Simple diffusion delivery via brain interstitial route for the treatment of cerebral ischemia. Sci. China Life Sci. 54: 235-239.

Hansen, A.J. (1985). Effect of anoxia on ion distribution in the brain. Physiol. Rev. 65: 101-148.

Hladky, S.B. and Barrand, M.A. (2014). Mechanisms of fluid movement into, through and out of the brain: evaluation of the evidence. Fluids Barriers CNS 11: 26.

Hou, J., Wang, W., Quan, X., Liang, W., Li, Z., Chen, D., Han, H. (2017). Quantitative visualization of dynamic tracer transportation in the extracellular space of deep brain regions using tracer-based magnetic resonance imaging. Med. Sci. Monit. 23: 4260-4268.

Hrabetova, S., Cognet, L., Rusakov, D.A., Nagerl, U.V. (2018). Unveiling the extracellular space of the brain: from super-resolved microstructure to in vivo function. J. Neurosci. 38: 9355-9363.
Hrabetova, S. and Nicholson, C. (2007). Biophysical properties of brain extracellular space explored with ion-selective microelectrodes, integrative optical imaging and related techniques. In: Michael, A.C. and Borland, L.M., (Eds). Electrochemical methods for neuroscience. CRC Press/Taylor \& Francis, Boca Raton (FL).

Hsu, W., Lesniak, M.S., Tyler, B., Brem, H. (2005). Local delivery of interleukin-2 and adriamycin is synergistic in the treatment of experimental malignant glioma. J. Neuro. Oncol. 74: 135-140.

Ibrahim, M.A., Hazhirkarzar, B., Dublin, A.B. (2020). Magnetic resonance imaging (MRI) gadolinium. StatPearls, Treasure Island (FL).

Iliff, J.J. and Nedergaard, M. (2013). Is there a cerebral lymphatic system? Stroke 44: S93-S95.

Iliff, J.J., Wang, M., Liao, Y., Plogg, B.A., Peng, W., Gundersen, G.A., Benveniste, H., Vates, G.E., Deane, R., Goldman, S.A., et al. (2012). A paravascular pathway facilitates CSF flow through the brain parenchyma and the clearance of interstitial solutes, including amyloid beta. Sci. Transl. Med. 4: 147ra111.

Inavalli, V., Lenz, M.O., Butler, C., Angibaud, J., Compans, B., Levet, F., Tonnesen, J., Rossier, O., Giannone, G., Thoumine, O., et al. (2019). A super-resolution platform for correlative live singlemolecule imaging and STED microscopy. Nat. Methods 16: $1263-1268$.

Iozzo, R.V. (1998). Matrix proteoglycans: from molecular design to cellular function. Annu. Rev. Biochem. 67: 609-652.

Jain, R.K. (1987). Transport of molecules in the tumor interstitium: a review. Canc. Res. 47: 3039-3051.

Karlsson, U. and Schultz, R. (1964). Plasma membrane apposition in the central nervous system after aldehyde perfusion. Nature 201: 1230-1231.

Katzman, R. (1961). Electrolyte distribution in mammalian central nervous system. Are glia high sodium cells? Neurology 11: 27-36.

Korogod, N., Petersen, C.C., Knott, G.W. (2015). Ultrastructural analysis of adult mouse neocortex comparing aldehyde perfusion with cryo fixation. Elife 4: e05793.

Kostka, L., Kotrchova, L., Subr, V., Libanska, A., Ferreira, C.A., Malatova, I., Lee, H.J., Barnhart, T.E., Engle, J.W., Cai, W., et al. (2020). HPMA-based star polymer biomaterials with tuneable structure and biodegradability tailored for advanced drug delivery to solid tumours. Biomaterials 235: 119728.

Krishnaswamy, V.R., Benbenishty, A., Blinder, P., Sagi, I. (2019). Demystifying the extracellular matrix and its proteolytic remodeling in the brain: structural and functional insights. Cell. Mol. Life Sci. 76: 3229-3248.

Lehmenkuhler, A., Sykova, E., Svoboda, J., Zilles, K., Nicholson, C. (1993). Extracellular space parameters in the rat neocortex and subcortical white matter during postnatal development determined by diffusion analysis. Neuroscience 55: 339-351.

Lei, Y., Han, H., Yuan, F., Javeed, A., Zhao, Y. (2017). The brain interstitial system: anatomy, modeling, in vivo measurement, and applications. Prog. Neurobiol. 157: 230-246.

Lenz, M.O., Sinclair, H.G., Savell, A., Clegg, J.H., Brown, A.C., Davis, D.M., Dunsby, C., Neil, M.A., French, P.M. (2014). 3-D stimulated emission depletion microscopy with programmable aberration correction. J. Biophot. 7: 29-36.

Levin, V.A., Fenstermacher, J.D., Patlak, C.S. (1970). Sucrose and inulin space measurements of cerebral cortex in four mammalian species. Am. J. Physiol. 219: 1528-1533. 
Li, C., Liu, S., Wang, W., Liu, W., Kuang, C., Liu, X. (2018). Recent research on stimulated emission depletion microscopy for reducing photobleaching. J. Microsc. 271: 4-16.

Li, K., Han, H., Zhu, K., Lee, K., Liu, B., Zhou, F., Fu, Y., He, Q. (2013). Real-time magnetic resonance imaging visualization and quantitative assessment of diffusion in the cerebral extracellular space of C6 glioma-bearing rats. Neurosci. Lett. 543: 84-89.

Lin, R., Shi Ng, L., Wang, C.H. (2005). In vitro study of anticancer drug doxorubicin in PLGA-based microparticles. Biomaterials 26: 4476-4485.

Lundbaek, J.A. and Hansen, A.J. (1992). Brain interstitial volume fraction and tortuosity in anoxia. Evaluation of the ionselective micro-electrode method. Acta Physiol. Scand. 146: 473-484.

Mestre, H., Du, T., Sweeney, A.M., Liu, G., Samson, A.J., Peng, W., Mortensen, K.N., Staeger, F.F., Bork, P.A.R., Bashford, L., et al. (2020). Cerebrospinal fluid influx drives acute ischemic tissue swelling. Science 367: eaax7171.

Mu, L. and Feng, S.S. (2003). PLGA/TPGS nanoparticles for controlled release of paclitaxel: effects of the emulsifier and drug loading ratio. Pharmaceut. Res. 20: 1864-1872.

Murphy, T.R., Davila, D., Cuvelier, N., Young, L.R., Lauderdale, K., Binder, D.K., Fiacco, T.A. (2017). Hippocampal and cortical pyramidal neurons swell in parallel with astrocytes during acute hypoosmolar stress. Front. Cell. Neurosci. 11: 275.

Nicholson, C. (1993). Ion-selective microelectrodes and diffusion measurements as tools to explore the brain cell microenvironment. J. Neurosci. Methods 48: 199-213.

Nicholson, C. and Hrabetova, S. (2017). Brain extracellular space: the final Frontier of neuroscience. Biophys. J. 113: 2133-2142.

Nicholson, C. and Phillips, J.M. (1981). Ion diffusion modified by tortuosity and volume fraction in the extracellular microenvironment of the rat cerebellum. J. Physiol. 321: 225-257.

Nicholson, C. and Tao, L. (1993). Hindered diffusion of high molecular weight compounds in brain extracellular microenvironment measured with integrative optical imaging. Biophys. J. 65: 2277-2290.

Nordling-David, M.M., Yaffe, R., Guez, D., Meirow, H., Last, D., Grad, E., Salomon, S., Sharabi, S., Levi-Kalisman, Y., Golomb, G., et al. (2017). Liposomal temozolomide drug delivery using convection enhanced delivery. J. Contr. Release 261: 138-146.

Odackal, J., Colbourn, R., Odackal, N.J., Tao, L., Nicholson, C., Hrabetova, S. (2017). Real-time iontophoresis with tetramethylammonium to quantify volume fraction and tortuosity of brain extracellular space. J. Vis. Exp. 125: 55755.

Patlak, C.S. and Fenstermacher, J.D. (1975). Measurements of dog blood-brain transfer constants by ventriculocisternal perfusion. Am. J. Physiol. 229: 877-884.

Perkins, K.L., Arranz, A.M., Yamaguchi, Y., Hrabetova, S. (2017). Brain extracellular space, hyaluronan, and the prevention of epileptic seizures. Rev. Neurosci. 28: 869-892.

Plog, B.A., Lou, N., Pierre, C.A., Cove, A., Kenney, H.M., Hitomi, E., Kang, H., Iliff, J.J., Zeppenfeld, D.M., Nedergaard, M., et al. (2019). When the air hits your brain: decreased arterial pulsatility after craniectomy leading to impaired glymphatic flow. J. Neurosurg. 133: 1-14.

Reardon, D.A., Rich, J.N., Friedman, H.S., Bigner, D.D. (2006). Recent advances in the treatment of malignant astrocytoma. J. Clin. Oncol. 24: 1253-1265.
Ren, R., Shi, C., Cao, J., Sun, Y., Zhao, X., Guo, Y., Wang, C., Lei, H., Jiang, H., Ablat, N., et al. (2016). Neuroprotective effects of a standardized flavonoid extract of safflower against neurotoxininduced cellular and animal models of Parkinson's disease. Sci. Rep. 6: 22135.

Reum, T., Olshausen, F., Mazel, T., Vorisek, I., Morgenstern, R., Sykova, E. (2002). Diffusion parameters in the striatum of rats with 6-hydroxydopamine-induced lesions and with fetal mesencephalic grafts. J. Neurosci. Res. 70: 680-693.

Reynaud, O., Winters, K.V., Hoang, D.M., Wadghiri, Y.Z., Novikov, D.S., Kim, S.G. (2016). Pulsed and oscillating gradient MRI for assessment of cell size and extracellular space (POMACE) in mouse gliomas. NMR Biomed. 29: 1350-1363.

Roitbak, T. and Sykova, E. (1999). Diffusion barriers evoked in the rat cortex by reactive astrogliosis. Glia 28: 40-48.

Rosenberg, G.A., Kyner, W.T., Estrada, E. (1980). Bulk flow of brain interstitial fluid under normal and hyperosmolar conditions. Am. J. Physiol. 238: F42-F49.

Rungta, R.L., Choi, H.B., Tyson, J.R., Malik, A., Dissing-Olesen, L., Lin, P.J.C., Cain, S.M., Cullis, P.R., Snutch, T.P., MacVicar, B.A. (2015). The cellular mechanisms of neuronal swelling underlying cytotoxic edema. Cell 161: 610-621.

Saghyan, A., Lewis, D.P., Hrabe, J., Hrabetova, S. (2012). Extracellular diffusion in laminar brain structures exemplified by hippocampus. J. Neurosci. Methods 205: 110-118.

Saini, M., Roser, F., Hussein, S., Samii, M., Bellinzona, M. (2004). Intralesional mitoxantrone biopolymer-mediated chemotherapy prolongs survival in rats with experimental brain tumors. J. Neuro. Oncol. 68: 225-232.

Sawyer, A.J., Piepmeier, J.M., Saltzman, W.M. (2006). New methods for direct delivery of chemotherapy for treating brain tumors. Yale J. Biol. Med. 79: 141-152.

Shi, M., Anantha, M., Wehbe, M., Bally, M.B., Fortin, D., Roy, L.O., Charest, G., Richer, M., Paquette, B., Sanche, L. (2018). Liposomal formulations of carboplatin injected by convectionenhanced delivery increases the median survival time of F98 glioma bearing rats. J. Nanobiotechnol. 16: 77.

Song, G., Luo, T., Dong, L., Liu, Q. (2017). Extracellular diffusion quantified by magnetic resonance imaging during rat $\mathrm{C} 6$ glioma cell progression. Braz. J. Med. Biol. Res. 50: e5403.

Soria, F.N., Paviolo, C., Doudnikoff, E., Arotcarena, M.L., Lee, A., Danne, N., Mandal, A.K., Gosset, P., Dehay, B., Groc, L., et al. (2020). Synucleinopathy alters nanoscale organization and diffusion in the brain extracellular space through hyaluronan remodeling. Nat. Commun. 11: 3440.

Spector, R., Robert Snodgrass, S., Johanson, C.E. (2015). A balanced view of the cerebrospinal fluid composition and functions: focus on adult humans. Exp. Neurol. 273: 57-68.

Stachniak, T.J., Sylwestrak, E.L., Scheiffele, P., Hall, B.J., Ghosh, A. (2019). Elfn1-induced constitutive activation of mGluR7 determines frequency-dependent recruitment of somatostatin interneurons. J. Neurosci. 39: 4461-4474.

Sykova, E. (1983). Extracellular $\mathrm{K}^{+}$accumulation in the central nervous system. Prog. Biophys. Mol. Biol. 42: 135-189.

Sykova, E. (2004). Diffusion properties of the brain in health and disease. Neurochem. Int. 45: 453-466.

Sykova, E., Mazel, T., Simonova, Z. (1998). Diffusion constraints and neuron-glia interaction during aging. Exp. Gerontol. 33: 837-851. 
Sykova, E. and Nicholson, C. (2008). Diffusion in brain extracellular space. Physiol. Rev. 88: 1277-1340.

Sykova, E., Svoboda, J., Polak, J., Chvatal, A. (1994). Extracellular volume fraction and diffusion characteristics during progressive ischemia and terminal anoxia in the spinal cord of the rat. J. Cerebr. Blood Flow Metabol. 14: 301-311.

Sykova, E., Vorisek, I., Antonova, T., Mazel, T., Meyer-Luehmann, M., Jucker, M., Hajek, M., Ort, M., Bures, J. (2005). Changes in extracellular space size and geometry in APP23 transgenic mice: a model of Alzheimer's disease. Proc. Natl. Acad. Sci. U.S.A. 102: 479-484.

Teng, Z., Wang, A., Wang, P., Wang, R., Wang, W., Han, H. (2018). The effect of aquaporin-4 knockout on interstitial fluid flow and the structure of the extracellular space in the deep brain. Aging Dis. 9: 808-816.

Tomioka, N.H., Yasuda, H., Miyamoto, H., Hatayama, M., Morimura, N., Matsumoto, Y., Suzuki, T., Odagawa, M., Odaka, Y.S., Iwayama, Y., et al. (2014). Elfn1 recruits presynaptic mGluR7 in trans and its loss results in seizures. Nat. Commun. 5: 4501.

Tonnesen, J., Inavalli, V., Nagerl, U.V. (2018). Super-resolution imaging of the extracellular space in living brain tissue. Cell 172: 1108-1121 e1115

Valenzuela, J.C., Heise, C., Franken, G., Singh, J., Schweitzer, B., Seidenbecher, C.I., Frischknecht, R. (2014). Hyaluronan-based extracellular matrix under conditions of homeostatic plasticity. Philos. Trans. R. Soc. Lond. B Biol. Sci. 369: 20130606.

Van Harreveld, A. and Steiner, J. (1970). The magnitude of the extracellular space in electron micrographs of superficial and deep regions of the cerebral cortex. J. Cell Sci. 6: 793-805.

Vanharreveld, A., Crowell, J., Malhotra, S.K. (1965). A study of extracellular space in central nervous tissue by freezesubstitution. J. Cell Biol. 25: 117-137.

Vargova, L., Homola, A., Zamecnik, J., Tichy, M., Benes, V., Sykova, E. (2003). Diffusion parameters of the extracellular space in human gliomas. Glia 42: 77-88.

Wang, A., Wang, R., Cui, D., Huang, X., Yuan, L., Liu, H., Fu, Y., Liang, L., Wang, W., He, Q., et al. (2019). The drainage of interstitial fluid in the deep brain is controlled by the integrity of myelination. Aging Dis. 10: 937-948.

Wang, Y., Fehlhaber, K.E., Sarria, I., Cao, Y., Ingram, N.T., Guerrero-Given, D., Throesch, B., Baldwin, K., Kamasawa, N.,
Ohtsuka, T., et al. (2017). The auxiliary calcium channel subunit $\alpha 2 \delta 4$ is required for axonal elaboration, synaptic transmission, and wiring of rod photoreceptors. Neuron 93: 1359-1374 e1356.

Welsher, K., Liu, Z., Sherlock, S.P., Robinson, J.T., Chen, Z., Daranciang, D., Dai, H. (2009). A route to brightly fluorescent carbon nanotubes for near-infrared imaging in mice. Nat. Nanotechnol. 4: 773-780.

Wildanger, D., Medda, R., Kastrup, L., Hell, S.W. (2009). A compact STED microscope providing 3D nanoscale resolution. J. Microsc. 236: 35-43.

Xie, L., Kang, H., Xu, Q., Chen, M.J., Liao, Y., Thiyagarajan, M., O’Donnell, J., Christensen, D.J., Nicholson, C., Iliff, J.J., et al. (2013). Sleep drives metabolite clearance from the adult brain. Science 342: 373-377.

Xu, F., Hongbin, H., Yan, J., Chen, H., He, Q., Xu, W., Zhu, N., Zhang, H., Zhou, F., Lee, K. (2011). Greatly improved neuroprotective efficiency of citicoline by stereotactic delivery in treatment of ischemic injury. Drug Deliv. 18: 461-467.

Yamaguchi, Y. (2000). Lecticans: organizers of the brain extracellular matrix. Cell. Mol. Life Sci. 57: 276-289.

Yang, S., Wang, Y., Li, K., Tang, X., Zhang, K., Shi, C., Han, H., Peng, Y. (2016). Extracellular space diffusion analysis in the infant and adult rat striatum using magnetic resonance imaging. Int. J. Dev. Neurosci. 53: 1-7.

Yue, X., Mei, Y., Zhang, Y., Tong, Z., Cui, D., Yang, J., Wang, A., Wang, R., Fei, X., Ai, L., et al. (2019). New insight into Alzheimer's disease: light reverses Abeta-obstructed interstitial fluid flow and ameliorates memory decline in APP/PS1 mice. Alzheim. Demen. 5: 671-684.

Zamecnik, J., Vargova, L., Homola, A., Kodet, R., Sykova, E. (2004). Extracellular matrix glycoproteins and diffusion barriers in human astrocytic tumours. Neuropathol. Appl. Neurobiol. 30: 338-350.

Zimmermann, D.R. and Dours-Zimmermann, M.T. (2008). Extracellular matrix of the central nervous system: from neglect to challenge. Histochem. Cell Biol. 130: 635-653.

Zoremba, N., Homola, A., Rossaint, R., Sykova, E. (2007). Brain metabolism and extracellular space diffusion parameters during and after transient global hypoxia in the rat cortex. Exp. Neurol. 203: 34-41. 